\title{
Fluorescence Lifetime Imaging Microscopy of Amyloid Aggregates
}

\author{
Christine J. Koh and Minyung Lee*
}

\author{
Department of Chemistry and Division of Nanosciences, Ewha Womans University, Seoul 120-750, Korea \\ E-mail:mylee@ewha.ac.kr \\ Received December 19, 2005
}

Key Words : Amyolid, Aggregation, Inhibitor, Oligomer, FLIM

Amyloid hypothesis states that a chronic imbalance between $\beta$-amyloid(A $\beta$ ) peptide production and clearance causes $\mathrm{A} \beta$ deposition in the brain, neuronal death, and eventually Alzheimer's disease (AD). ${ }^{1-3}$ The physiological role of $\mathrm{A} \beta$ as well as its precursor, amyloid precursor protein (APP), is unknown. In abnormal metabolic processes, $\mathrm{A} \beta$ monomers associate further in vivo, forming dimers, multimers, or larger oligomers. Oligomers grow to higher order aggregates such as protofilaments and fibrils, leading to gradual insoluble plaque formation. Numerous reports indicate that $\mathrm{A} \beta$ oligomers are found to be highly toxic to neurons rather than senile plaques. These oligomers, also called as $\mathrm{A} \beta$-derived diffusible ligands (ADDL), has a spherical shape and its existence in vivo correlates well with dementia. ${ }^{4,5}$

In this work, we report a new oligomer probe, FL-A $\beta_{11-25^{-}}$ Azulene, utilizing the concept of FRET amyloid (Fig. 1). $\mathrm{FL}$, standing for fluorescein, is used as the fluorescence donor, azulene the energy acceptor, and the spacer $\mathrm{A} \beta_{11-25}$ that derives from the aggregation motive. ${ }^{6,7}$ The aggregation probe is an improved version of our previous one in a sense that the synthetic method is simpler. ${ }^{8}$ That is, azulenyl alanine as well as FL can be directly incorporated into the FRET probe in the course of solid peptide synethsis. The Förster distance $\mathrm{R}_{0}$ of fluorescein and azulene pair was calculated as $21 \AA$. We carried out the experiments at $\mathrm{pH} 5$ condition since amyloid aggregation has high possibility to occur at lysosomes. Figure 2 shows the change of the fluorescence decay of FL in FRET amyloid as a function of unlabeled amyloid $\mathrm{A} \beta_{11-25}$. The Figure also shows that critical micellar concentration (CMC) for amyloid aggregation becomes around $3 \mu \mathrm{M}$ in $\mathrm{pH} 5$. Amyloid aggregation

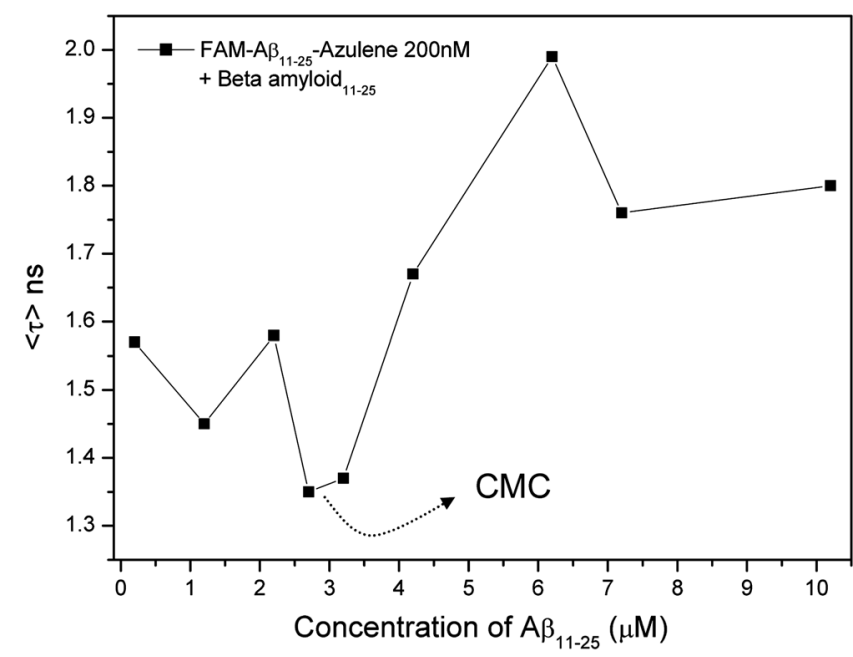

Figure 2. Fluorescence average lifetimes of Fluorescein, the energy donor as a function of unlabeled amyloid peptide concentration ( $\mathrm{pH} 5.0)$.
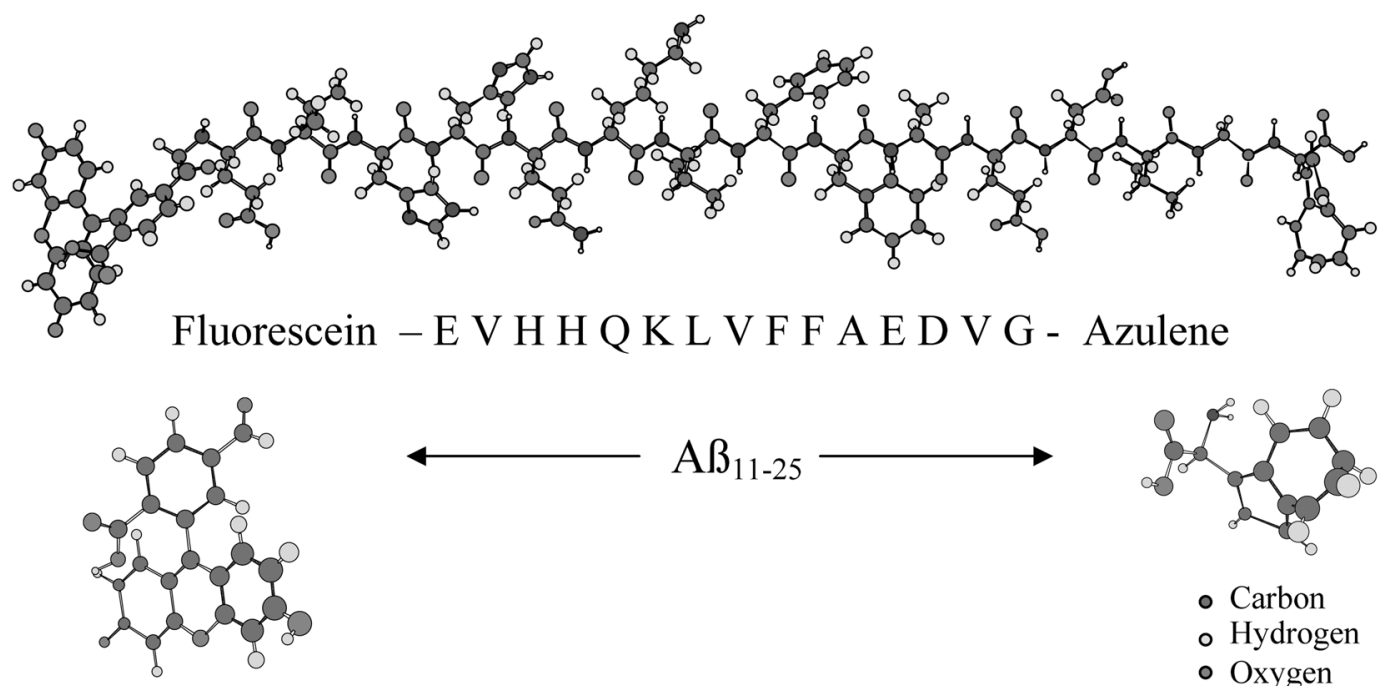

- Carbon

- Hydrogen

- Oxygen

- Nitrogen

Figure 1. FRET amyloid probe. 5-(and-6)-carboxyfluorescein-A $\beta_{11-25}$-Azulene. 


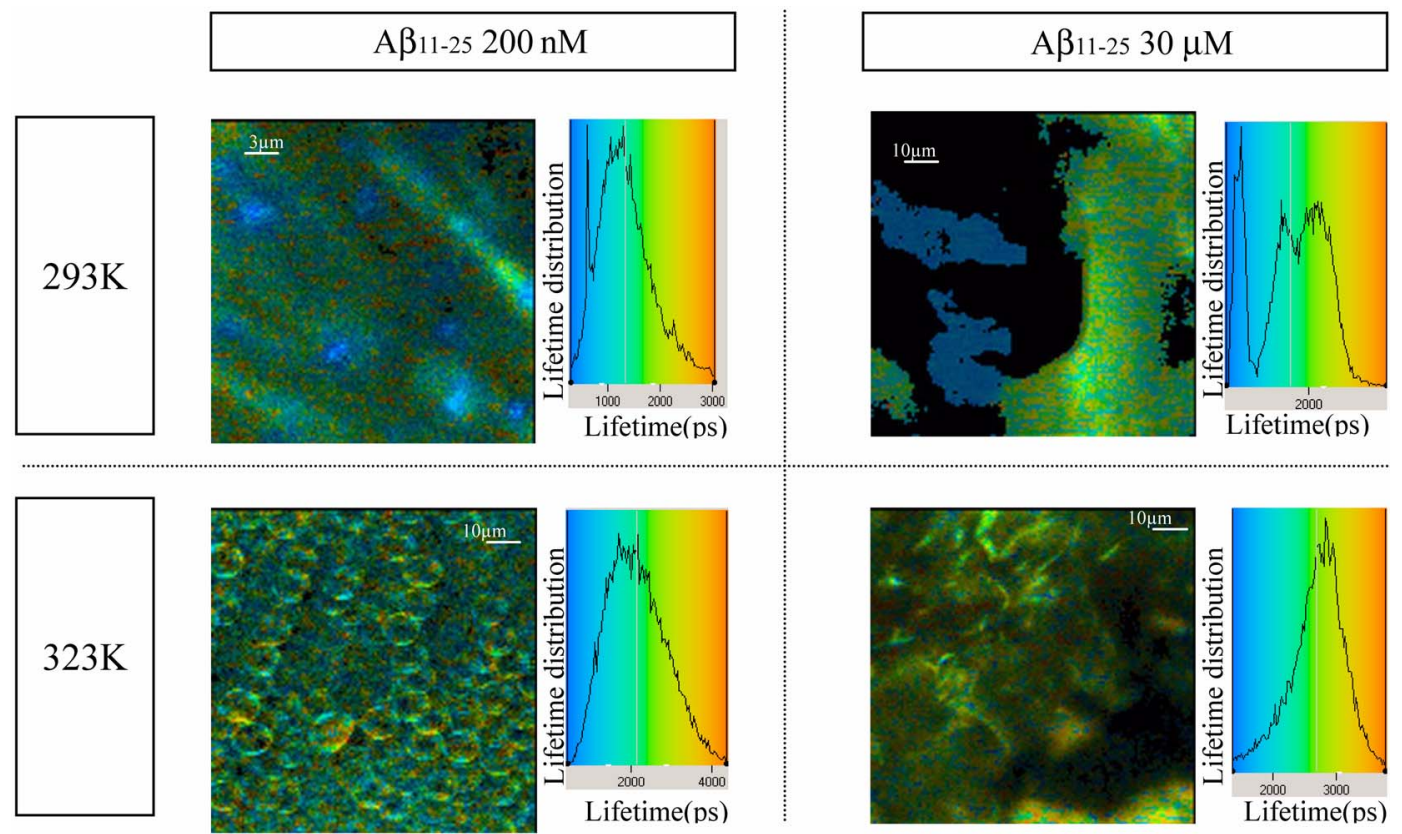

Figure 3. Fluorescence Lifetime Image of FRET amyloid probe with various condition in acetate buffer (pH 5.0).

was more pronounced in acidic rather than in neutral condition. These results suggest that FRET amyloid indicates the multi-step conformational changes of $\mathrm{A} \beta_{11-25}$ such as random coil, micellar structure, and extended $\beta$-sheet in fibrils. Owing to conformation switching, the FRET efficiency of the probe increases in the oligomer structure, thus the presence of oligomers can be probed by the fluorescence lifetime measurement and time-resolved confocal imaging.

For lifetime imaging, samples were prepared by dissolving amyloid peptides in DMSO to a final concentration in $\mathrm{NaAc}$ (pH 5.0) buffers with a few minute sonication. Buffers were filtrated with $0.22 \mu \mathrm{m}$ pore filter before use. Amyloid peptide solution of certain concentration with FRET amyloid probe was desiccated for 2-3 days at constant pressure and the temperature of $293 \mathrm{~K}$ or $343 \mathrm{~K}$ on cover glass. The sample was excited by $467 \mathrm{~nm}$ from picosecond pulsed diode laser (PicoQuant: PDL 800-B) operating at 20 $\mathrm{MHz}$ frequency and the emitted photons were detected by MCP-PMT (Hamamatsu 3809-07). Emission for the FL was detected at the wavelength from $506 \mathrm{~nm}$ to $560 \mathrm{~nm}$. The fluorescence decay curves were collected by a SPC-830 board (Becker-Hickl) synchronized with an AFM scanner (PSIA). Figure 3 shows the fluorescence lifetime imaging at $\mathrm{pH} 5$ in different concentration $(200 \mathrm{nM}$ and $30 \mu \mathrm{M})$ and temperatures ( $293 \mathrm{~K}$ and $323 \mathrm{~K}$ ). The solution contained the 10 nanomolar concentration of FRET amyloid as a probe. The lifetime imaging data clearly shows that the existence of micellar structure that appears as the short lifetime component ( $c a .600 \mathrm{ps}$ ). The population is more significant at the higher concentration. As the temperature rises, the short lifetime component disappears, indicating that the high temperature promotes the $\beta$-sheet aggregate formation. This is because the amyloid peptides are strongly hydrophobic. The data also shows a new form of amyloids resembling a donut-shape.

In conclusion, we presented a newly developed amyloid oligomer probe, FL-A $\beta_{11-25}$-Azulene and carried out the fluorescence lifetime imaging microscopy by utilizing its characteristics. The application of FLIM to amyloid aggregation process shows the oligomeric structures as well as a peculiar donut structure with very high sensitivity. These observations to probe the micellar structures are very meaningful, because it is usually difficult to distinguish micelle and protofibril structure with other techniques. The amyloid oligomer marker can be utilized in a single cell imaging for unraveling amyloid aggregation processes.

Acknowledgement. This work has been supported by a grant from Korea Research Foundation (2005).

\section{References}

1. Hardy, J.; Higgins, G. A. Science 1992, 256, 184.

2. Hardy, J.; Selkoe, D. J. Science 2000, 297, 353.

3. See, for example, Amyloid, Prions, and Other Protein Aggregates, Methods in Enzymology; Wetzel, R., Ed.; 1999; Vol. 309.

4. Lambert, M. P.; Barlow, A. K.; Chromy, B. A.; Edwards, C.; Freed, R.; Liosatos, M.; Morgan, T. E.; Rozovsky, I.; Trommer, B.; Viola, K. L.; Wals, P.; Zhang, C.; Finch, C. E.; Kraft, G. A.; Klein, W. Proc. Natl. Acad. Sci., USA 1998, 95, 6448.

5. Klein, W. L.; Krafft, G. A.; Finch, C. E. Trends in Neurosci. 2001, 24, 219.

6. Balbach, J. J.; Isshii, Y.; Antzutkin, O. N.; Leapman, R. D.; Rizzo, N. W.; Dyda, F.; Reed, J.; Tycko, R. Biochemistry 2000, 39, 13748 .

7. Walsh, D. M.; Tseng, B. P.; Rydel, R. E.; Podlisny, M. B.; Selkoe, D. J. Biochemistry 2000, 39, 10831.

8. Tjernberg, L. O.; Naslund, J.; Lindqvist, F.; Johansson, J.; Karlstrom, R.; Thyberg, J.; Terenius, L.; Nordstedt, C. J. Biol. Chem. 1996, 271, 8545 .

9. Kim, J.; Lee, M. Biochem. Biophys. Res. Comm. 2004, 316, 393. 